\title{
The H2020 European Project CLONETS: clock services over optical-fibre networks in Europe
}

\author{
Davide Calonico, Cecilia Clivati, Filippo Levi \\ Istituto Nazionale di Ricerca Metrologica, \\ Turin, Italy \\ d.calonico@inrim.it
}

\section{Przemysław Krehlik, Łukasz Śliwczyński AGH University of Science and Technology Cracow, Poland}

Jiri Dostal, Jan Radil, Vladimir Smotlacha,

$$
\begin{gathered}
\text { Radek Velc, Josef Vojtěch } \\
\text { CESNET } \\
\text { Prague, Czech Republic } \\
\\
\text { Mauro Campanella } \\
\text { Consortium GARR } \\
\text { Rome, Italy }
\end{gathered}
$$

Ondřej Č́ṕp, Simon Rerucha

Institute of Scientific Instruments of the ASCR, v.v.i. (ISI) Brno, Czech Republic

Ronald Holzwarth, Maurice Lessing, Sarah Saint-Jalm Menlo Systems GmbH Martinsried, Germany

Fabiola Camargo, Bruno Desruelle, Jean Lautier-Gaud Muquans

Talence, France

\section{Elizabeth Laier English, Jochen Kronjäger, Peter Whibberley \\ National Physical Laboratory \\ Teddington, United Kingdom}

Fratišek John, Milan Šnajder, Jiří Štefl
OPTOKON a.s. Jihlava, Czech Republic

Paweł Nogaś, Robert Urbaniak Piktime Systems sp. z o.o. Poznan, Poland

Artur Binczewski, Wojbor Bogacki, Krzysztof Turza Poznan Supercomputing and Networking Center Poznan, Poland

Gesine Grosche, Harald Schnatz Physikalisch-Technische Bundesanstalt Braunschweig, Germany

Emilie Camisard, Nicolas Quintin GIP-Renater Paris, France

Javier Diaz, Trinidad García, Eduardo Ros Seven Solutions S.L. Granada, Spain

$$
\begin{aligned}
& \text { Alessandro Galardini } \\
& \text { Conzorzio ToP-IX } \\
& \text { Turin, Italy }
\end{aligned}
$$

Alwyn Seeds, Zhen Yang University College London, United Kingdom

Anne Amy-Klein

LPL, Université Paris 13, CNRS

Villetaneuse, France

Eva Bookjans, Paul-Eric Pottie, and Philip Tuckey

LNE-SYRTE, Observatoire de Paris, PSL Research University, CNRS, Sorbonne Université

Paris, France

\footnotetext{
Abstract - The H2020 project CLONETS (www.clonets.eu) is a coordination and support action started in January 2017, and it aims at accelerating the take-up of the fibre link technology by indus-try, research and society. One of the main target is to
}

prepare the deployment of a permanent pan-European fibre network, providing high performance clock services to European re-search, and supporting wider services to industry and society. We report an update of the project. 


\section{Keywords-fiber link, T/F dissemination}

\section{INTRODUCTION}

Time and frequency transfer based on optical fibre links techniques have demonstrated excel-lent performances. They are a key technology for very demanding application in metrology and science, e.g. optical clock comparisons, and also for applications in industry and society. They are complementary and/or alternative to radio- and satellite-based transfer.

The $-\mathrm{H} 2020$ project CLONETS (www.clonets.eu) brings together National Metrology Institutes (NMIs), academic research groups, National Research and Education Network providers (NRENs), an internet exchange and small and medium-sized high-technology companies.

The main objectives include collecting information on needs, studying industrial and societal applications, defining strategies to support future work, training, focusing on contractual aspects.

Here, we present the advances on the different objectives. In particular, we describe (i) the first outcomes from a survey of needs and applications for time and frequency transfer over optical fibre, aimed at research infrastructures (ii) the presentation of a university master program (post-graduate level) and the short training courses. The survey activity will allow a better decision process about the future infrastructure. The training activity is intended to gain new competences useful for optical T/F metrology over fibre and to offer to scientists, metrologists, industry, society and research the opportunity of focusing on needs and possibilities presently available.

\section{HIGH PRECISION T/F NEEDS OF RESEARCH INFRASTRUCTURESE}

The availability of high performance time \& frequency $(\mathrm{T} / \mathrm{F})$ signals is at the very heart of research activities in fundamental physics, such as high-resolution spectroscopy, special and general relativity, the search for temporal variations of fundamental constants and quantum optics. Additionally, it will have a strong impact on a wide range of applied sciences, such as geodesy (chronometric levelling), Very Long Baseline Interferometry (VLBI) and laser ranging. The industrial applications of high performance $\mathrm{T} / \mathrm{F}$ signals are not limited to telecommunication (i.e. the supervision of the next generation of mobile networks), but will also include applications such as high-speed trading, synchronisation in smart grids and the augmentation of existing techniques of the Global Navigation Satellite Systems (GNSS) such as Global Positioning System (GPS), or Galileo. In the future, high performance T/F signals derived from ultra-stable lasers or optical clocks will play a similarly important role in the development of novel applications and fundamental research as low noise RFoscillators and primary Cs clocks have done over the last decades.

Today's best optical clocks can reach an estimated systematic fractional frequency uncertainty at the level of a few 10-18 ([1-3]). Satellite-based frequency comparison techniques offer world-wide coverage, but lack the performance required for optical clock comparisons (e.g. [4]). A transportable clock has become available recently $([5,6])$, but with an accuracy of $7 \times 10^{-17}$ it is still far from the full performance of its stationary counterparts. The unprecedented accuracy of modern optical clocks has spurred the development frequency comparison techniques with an equivalent performance. An optical frequency transfer over fibre is currently the only technique capable of comparing high-performance clocks across long distances without degrading the stability and accuracy of the comparison ([7, 8]). In the European Union (EU) funded projects NEAT-FT and OFTEN of the European Metrology and Research Programme (EMRP) and its successor European Metrology Programme for Innovation and Research (EMPIR), technologies for disseminating and comparing ultra-stable optical and radio-frequencies over fibre links have been demonstrated. The efforts of these projects have culminated in the first international comparison of primary Cs fountains and optical clocks, which was not limited by the link between the clocks $([9,10])$.

Although an optical fibre frequency transfer is capable of reaching a stability and an accuracy in the $10^{-19}$ to $10^{-20}$ range $[11,12]$, the fibre-based frequency transfer capabilities in Europe have not yet advanced towards the development of a sustainable, universal tool for $\mathrm{T} / \mathrm{F}$ metrology for Europe. Creating a $\mathrm{T} / \mathrm{F}$ infrastructure benefitting European society, the main issues hampering this development need to be addressed. Firstly, there is neither a reliable data base describing the $\mathrm{T} / \mathrm{F}$ transfer techniques currently being used or evaluated by Research Infrastructures (RIs) nor is there a clear understanding of the current and future needs of such RIs for precise and accurate T/F transfers. Secondly, the capability and the benefits of fibre based $\mathrm{T} / \mathrm{F}$ services are largely unknown to the user community and thirdly, the capacity for the integration of an $\mathrm{T} / \mathrm{F}$ transfer in existing networks provided e.g. by National Research and Education Network (NRENs) is up to now unexplored.

This deliverable focuses on the needs of RIs for a high precision $\mathrm{T} / \mathrm{F}$ transfer, whereby high precision refers to performances superior to those provided by standard Precision Time Protocol (PTP), e.g currently served by H-masers, Rb clocks or GNSS. The report is based on data collected through a survey of RIs, which previously had been identified as relevant..

\section{A. Relevant Research Infrastructures}

Relevant RIs in the context of this deliverable are institutes and organizations, which have timing needs in excess of standard PTP, H-masers or GNSS based services. They include National Metrology Institutes (NMIs), university based research groups in the field of quantum optics, research organizations (e.g. academies of science, the Max-Planck Society and the Fraunhofer Gesellschaft) and certain pertinent consortia interested in sharing $\mathrm{T} / \mathrm{F}$ transfer technology (e.g. the ACES consortium, the Geodetic Observatory Wettzell and the International Association of Geodesy).

Additionally, big infrastructures according to European Strategy Forum on Research Infrastructures (ESFRI) were considered as relevant, in particular, RIs from PHYSICAL SCIENCES \& ENGINEERING [13], such as the European Extremely Large Telescope [14], the European X-Ray FreeElectron Laser [15], the Facility for Antiproton and Ion Research [16], the European Organization for Nuclear Research [17], the 
Square Kilometre Array [18], the European VLBI Network [19], the Joint Institute for VLBI ERIC [20], and the European Space Agency [21] were contacted.

In total, 52 RIs deemed as representative of the institutes and organizations having high precision timing needs were identified and contacted for the survey by means of an electronic questionnaire. Among these 52 RIs are 17 NMIs with the remaining 35 RIs being classified as non-NMIs. Previously established contacts to the relevant RIs were exploited to ensure an adequate response to the survey. Overall, 37 RIs (11 NMIs and 26 non-NMIs) responded to the survey, which is sufficient to obtain a general overview of the T/Fneeds of RIs. Fig.1-4 show some relevant information on the RI interviewed for the survey.

The RIs that participated in the survey are located throughout Europe with responses coming from a wide range of different countries (Figure 1). There is a relatively large participation in France and the Czech Republic. The NRENs in these countries are very active in the field Time \& Frequency distribution potentially leading to a greater awareness of the topic.

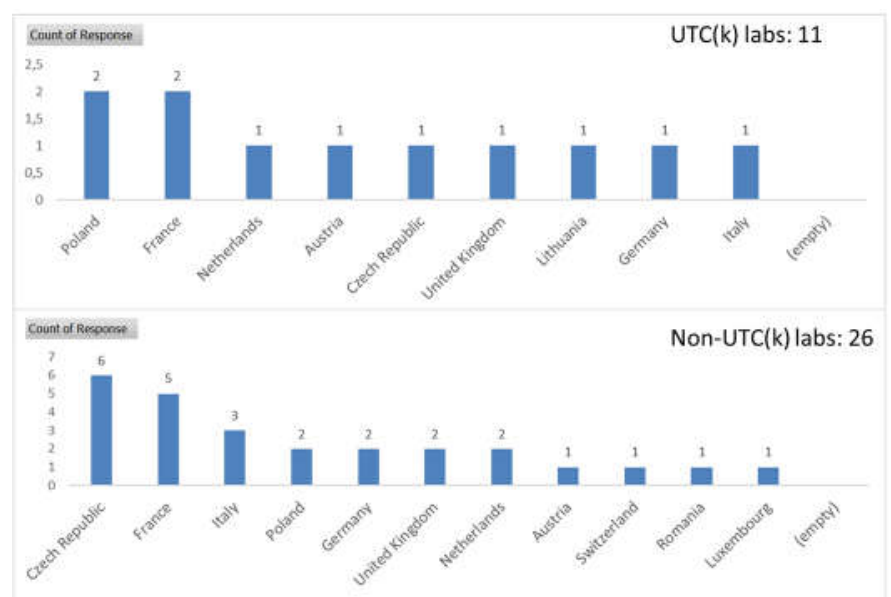

Fig. 1. Responses received by UTC(k) labs (top) and non-UTC(k) labs (bottom) according to county.

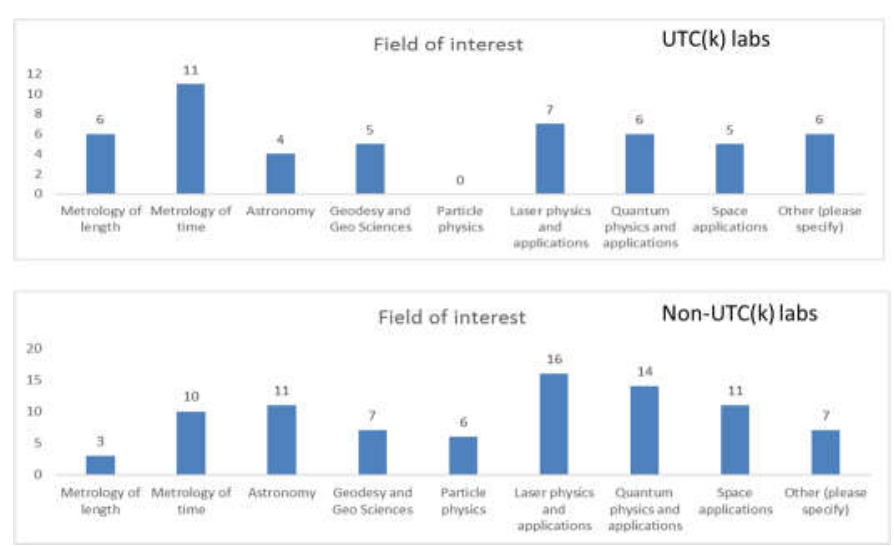

Fig. 2. Field of interest for UTC(k) and Non-UTC(k) labs.

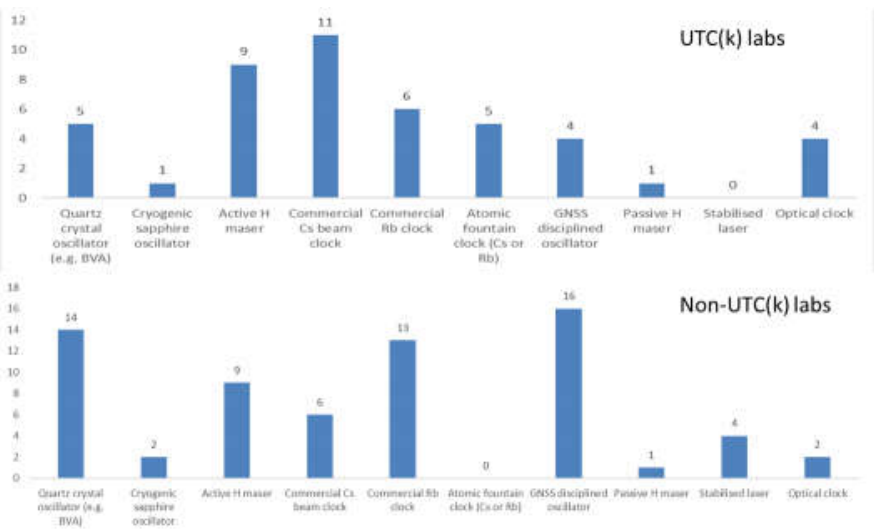

Fig. 3. Frequency standards and clocks operated at the surveyed UTC-labs (above) and non- UTC-labs (below).

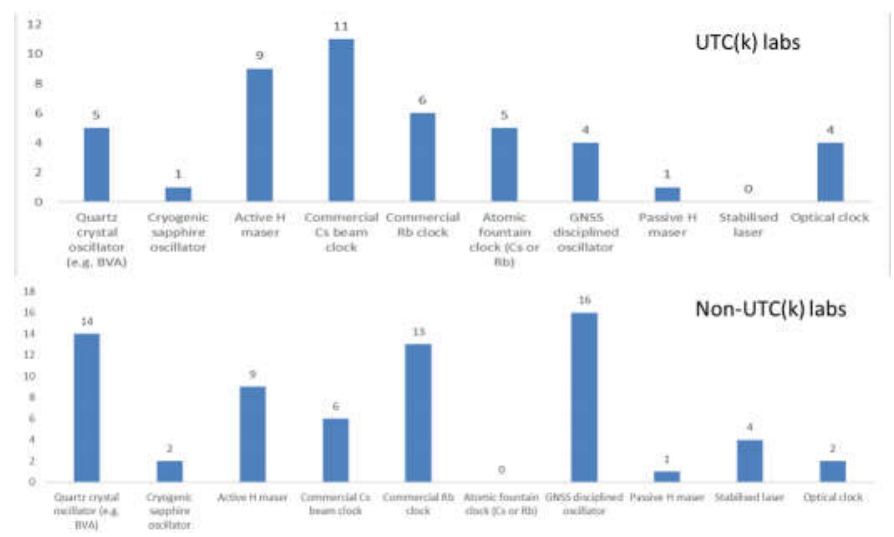

Fig. 4. Source of external T/Freference signals for UTC-labs (above) and nonUTC-labs (below).

\section{B. Results from the Survey}

The results of the survey are described in the Fig. 4 to 9. In general, the survey has revealed that applications requiring highest-performance frequency accuracy and instability prevail upon those requiring precise timing. As of today, receiving time is still based on satellite techniques. However, the most demanding timing applications already require an instability that can only be met by dissemination through optical fibres. Among the respondents there seems to be a clear view and ranking of the importance of traceability to the SI, the availability of $\mathrm{T} /$ Fsignals at a remote location, the resilience of such signals and issues related to security. The availability of a fibre based T/Fservice is considered as of highest importance for UTC labs as well as for non-UTC labs. While traceability is considered important (especially by $\mathrm{UTC}(\mathrm{k})$ labs), it is not regarded as particularly problematic by non-UTC labs. Considering that availability is rated highest, it is clear that resilience attracted significantly more consideration than security. Overall, the results of the survey support the CLONETS consortium's current understanding of the relevance of high precision $\mathrm{T} / \mathrm{F}$ reference signals for RIs. The communities that believe to benefit most from a fibre based $\mathrm{T} / \mathrm{F}$ service are - as expectedradio-astronomy, geodesy, accelerator- and spectroscopylaboratories, calibration laboratories and potentially space agencies. There is a growing awareness of the potential of a 
fibre-based T/F service and at the same time an increasing need for better performance than currently available via satellite based technology. The survey intended to identify the current and future needs of such research infrastructures for precise and accurate $\mathrm{T} / \mathrm{F}$ transfer. We received an adequate number of responses to consider the outcome of the survey as a representative spectrum of the high precision $\mathrm{T} / \mathrm{F}$ needs of European RIs. Together with the outcome of a survey performed in WP3 addressing the needs of European industry we will be able to shape a future time and frequency service that covers the needs of science, industry and society.
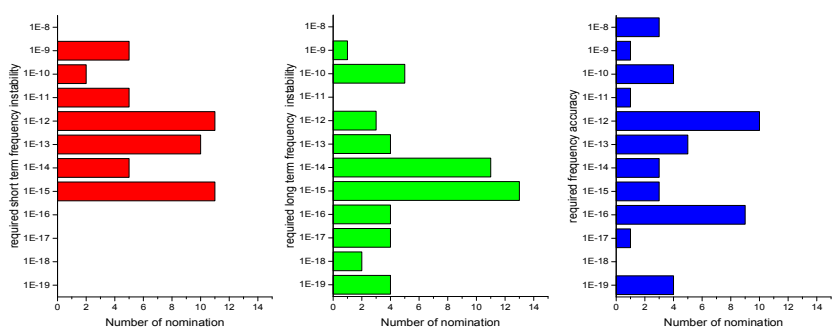

Fig. 5. Frequency instability required by RI
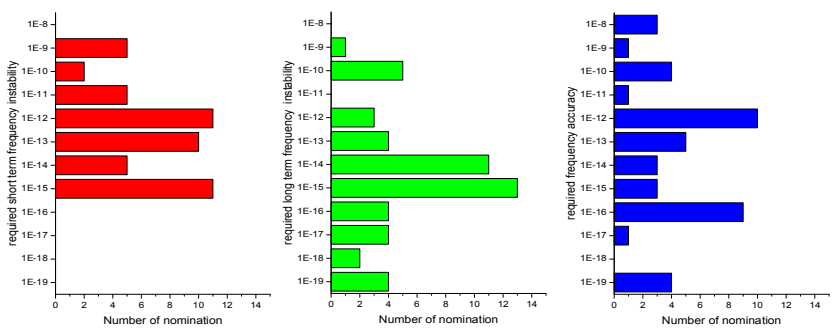

Fig. 6. Timing instability required by RI

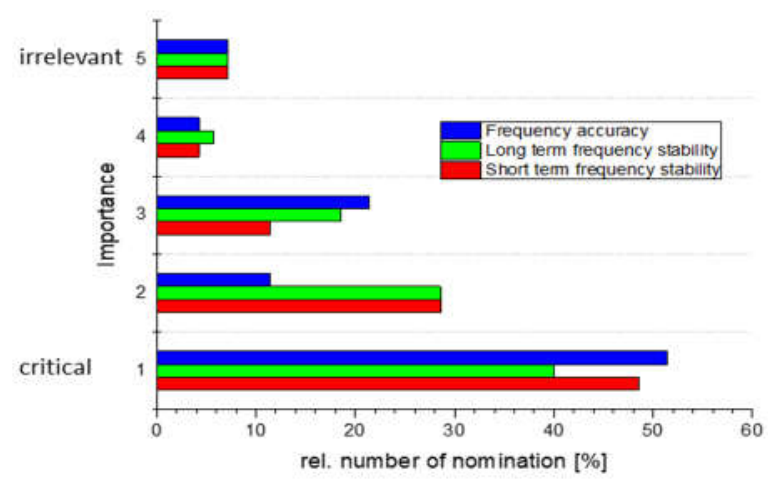

Fig. 7. Rating of the importance of frequency accuracy and instability

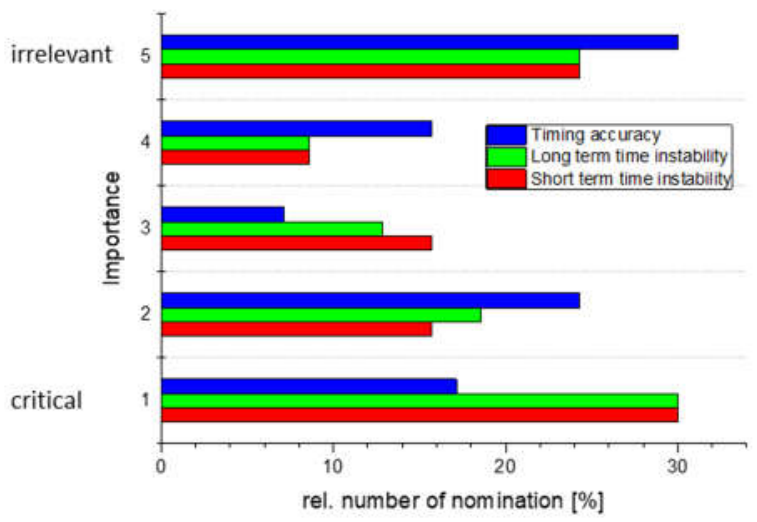

Fig. 8. Rating of the importance of timing accuracy and instability

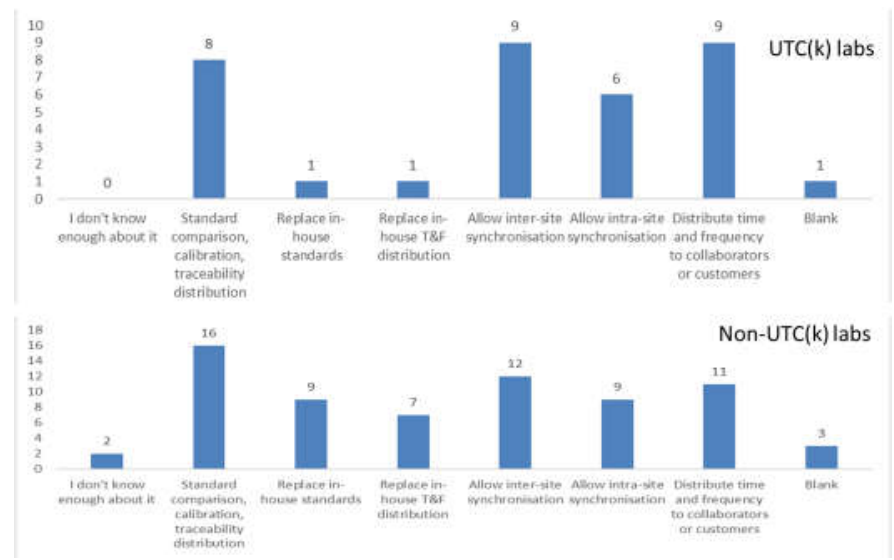

Fig. 9. Respones to the question: How could high performance T/Fdelivered over optical fibre open new possibilities that your organisation would be interested in?

\section{A SECOND LEVEL MASTER PROGRAMME}

\section{A. Motivations}

CLONETS organizes a second level master with the Politecnico of Turin, "Photonics for Data Networks and Metrology". Data traffic will experience a dramatic growth over the next years driven by $5 \mathrm{G}$ access, high-definition video, virtual and augmented-reality contents, and the considerable growth in cloud services due to Big Data Exchange. Photonic data networks will be required to be more and more pervasive and elastic, to supporting the paradigm of Internet of Thing and to enabling Industry 4.0. Besides data transport, photonic networks will also distribute time and frequency $(\mathrm{T} / \mathrm{F})$ standards for research and industry, enabling orders of magnitude performance improvements with respect to satellite systems, over continental geographical areas. The list of institutions supporting the initiative testifies the need for a vertical multidisciplinary knowledge, from the transmission layer up to the IP layer, and training such new professional figures is indeed the mission of the 2nd level Specializing Master's Programme. 
The Programme will offer theoretical and practical lecturing, hand-on experiences and a final internship in European industrial and/or research environments.

The purpose of the Programme is to give multidisciplinary knowledge and competence on photonics transmission and networking as well as on fundamentals of metrology and of time reference distribution on telecommunications infrastructure based on optical fiber transmission. The Programme is aimed at students with different backgrounds: graduated in the ICT field and graduated from schools of Physics and Metrology, and will train hybrid professional figures who will be able to design and manage state-of-the-art photonic networks to support the everincreasing IP traffic demands well as to distribute time references from and among metrology centers.

The 2nd level Specializing Master is a full time Programme of one academic year duration for a total of 69 European Credits Transfer System; it will start in September 2018 and will end in November 2019.

Lectures will be held in English at Politecnico di Torino premises - starting in September 2018 and ending in June 2019.

Lectures include classroom activities with academic and corporate teaching staff.

The internship period will start in July/August 2019 and will end in October/November 2019.

The teaching Programme is organized as follows:

i) courses: 32 weeks of full-time classroom activities (September 2018 - June 2019)

ii) internship: of on the job training at companies and/or research institutes in the photonic technologies field (July/August 2019 October/November 2019).

TABLE I. ERRORE. L'ORIGINE RIFERIMENTO NON È STATA TROVATA.COURSES AT THE CLONETS MASTER.

\begin{tabular}{|c|l|c|c|}
\hline n. & \multicolumn{1}{|c|}{ Subject } & Ects & Hours \\
\hline 1 & Digital Communication & 6 & 60 \\
\hline 2 & Optical Transmission & 5 & 50 \\
\hline 3 & Photonic Devices & 4 & 40 \\
\hline 4 & Time and frequency metrology & 5 & 50 \\
\hline 5 & Ultrabroadband access networks & 5 & 50 \\
\hline 6 & Long-haul optical transport & 4 & 40 \\
\hline 7 & Photonic Networks & 6 & 60 \\
\hline 8 & Quantum Communications & 4 & 40 \\
\hline 9 & $\begin{array}{l}\text { Photonics applications in } \\
\text { metrology }\end{array}$ & 4 & 40 \\
\hline 10 & Time and frequency laboratory & 6 & 60 \\
\hline 11 & Security for ICT & 4 & 40 \\
\hline 12 & Communications laboratory & 610 & 60 \\
\hline 13 & Internship & & \\
\hline
\end{tabular}

\section{ACKNOWLEDGMENT}

The Consortium thanks Prof. Vittorio Curri of the Politecnico of Torino for the organizion of the Master.

\section{REFERENCES}

[1] N. Huntemann, C. Sanner, B. Lipphardt, Chr. Tamm, and E. Peik. 'SingleIon Atomic Clock with $3 \times 10-18$ Systematic Uncertainty'. Physical Review Letters 116, no. 6 (8 February 2016): 063001. https://doi.org/10.1103/PhysRevLett.116.063001.

[2] T. L. Nicholson, S.L. Campbell, R.B. Hutson, G.E. Marti, B.J. Bloom, R.L. McNally, W. Zhang, et al. 'Systematic Evaluation of an Atomic Clock at $2 \times 10-18$ Total Uncertainty'. Nature Communications 6 (21 April 2015): 6896. https://doi.org/10.1038/ncomms7896.

[3] I. Ushijima, M. Takamoto, M. Das, T. Ohkubo, and H. Katori. 'Cryogenic Optical Lattice Clocks’. Nature Photonics 9 (9 February 2015): 185. https://doi.org/10.1038/nphoton.2015.5.

[4] S. Droste, C. Grebing, J. Leute, S. M. F. Raupach, A. Matveev, T. W. Hänsch, A. Bauch, R. Holzwarth, and G. Grosche. 'Characterization of a $450 \mathrm{Km}$ Baseline GPS Carrier-Phase Link Using an Optical Fiber Link'. New Journal of Physics 17, no. 8 (2015): 083044. https://doi.org/10.1088/1367-2630/17/8/083044.

[5] S. B. Koller, , J. Grotti, St. Vogt, A. Al-Masoudi, S. Dörscher, S. Häfner, U. Sterr, and Ch. Lisdat. 'Transportable Optical Lattice Clock with 7x1017 Uncertainty'. Physical Review Letters 118, no. 7 (13 February 2017): 073601. https://doi.org/10.1103/PhysRevLett.118.073601.

[6] J. Grotti, et al. 'Geodesy and Metrology with a Transportable Optical Clock'. Nature Physics, 12 February 2018. https://doi.org/10.1038/s41567-017-0042-3.

[7] R. Sebastian, A. Koczwara, and G. Grosche. 'Brillouin Amplification Supports 1x10-20 Uncertainty in Optical Frequency Transfer over 1400 $\mathrm{Km}$ of Underground Fiber'. Physical Review A 92, no. 2 (24 August 2015): 021801.

[8] C., Nicola, N. Quintin, F. Stefani, F. Wiotte, E. Camisard, C. Chardonnet, G. Santarelli, A. Amy-Klein, P. E. Pottie, and O. Lopez. 'Cascaded Optical Fiber Link Using the Internet Network for Remote Clocks Comparison'. Optics Express 23, no. 26 (28 December 2015): 33927-37. https://doi.org/10.1364/OE.23.033927.

[9] C. Lisdat, G. Grosche, N. Quintin, C. Shi, S.M.F. Raupach, C. Grebing, D. Nicolodi, et al. 'A Clock Network for Geodesy and Fundamental Science'. Nature Communications 7 (9 August 2016): 12443.

[10] .J Guéna, S Weyers, M Abgrall, C Grebing, V Gerginov, P Rosenbusch, $\mathrm{S}$ Bize, et al. 'First International Comparison of Fountain Primary Frequency Standards via a Long Distance Optical Fiber Link'. Metrologia 54, no. 3 (2017): 348. https://doi.org/10.1088/1681-7575/aa65fe.

[11] S. M. F. Raupach, A. Koczwara and G. Grosche Brillouin amplification supports 10-20 accuracy in optical frequency transfer over $1400 \sim \mathrm{km}$ of underground fibre, Phys. Rev. $\sim$ A 92, 021801(R) (2015)

[12] A. Bercy, O. Lopez, P.-E. Pottie and A. Amy-Klein Ultrastable optical frequency dissemination on a multi-access fibre network Appl. Phys. $\sim \mathrm{B}$ $122,189(2016)$

[13] https://ec.europa.eu/research/infrastructures/pdf/ri_landscape_2017.pdf\# view $=$ fit\&pagemode $=$ none

[14] http://www.eso.org/public/about-eso/faq/faq-elt/

[15] https://www.xfel.eu/

[16] http://www.fair-center.de/index.php?id=1\&L=1

[17] https://home.cern/

[18] https://www.skatelescope.org/

[19] http://www.evlbi.org/

[20] https://www.jive.nl/

[21] https://www.esa.int/ESA 